\title{
Inter-ethnic genetic variations and novel variant identification in the partial sequences of CYP2B6 gene in Pakistani population
}

\author{
Sagheer Ahmed ${ }^{1}$, Hizbullah Khan ${ }^{1}$, Asifullah Khan ${ }^{2}$, Muhammad Hanif Bangash ${ }^{3}$, Abrar Hussain ${ }^{4}$, Mughal \\ Qayum $^{5}$, Muhammad Hamid Hamdard ${ }^{\text {Corresp. } 6}$ \\ 1 Department of Basic Medical Sciences, Shifa College of Pharmaceutical Sciences, Shifa Tameer-e-Millat University, Islamabad, Pakistan \\ 2 Department of Biochemistry, Abdul Wali Khan University, Mardan, Pakistan \\ 3 Pakistan Institute of Nuclear Science \& Technology, Islamabad, Pakistan \\ 4 Baluchistan University of Information Technology and Management Sciences, Quetta, Pakistan \\ 5 Department of Pharmacy, Kohat University of Science \& Technology, Kohat, Pakistan \\ 6 Faculty of Biology, Kabul University, Kabul, Afghanistan \\ Corresponding Author: Muhammad Hamid Hamdard \\ Email address: hamid.hamdard@ku.edu.af
}

Background: Some single nucleotide polymorphisms (SNPs) in the cytochrome P450 (CYP)2B6 gene lead to decreased enzyme activity and have an impact on drug metabolism. The present study was designed to investigate the patterns of genetic distinction across a hypervariable region of the CYP2B6 gene, known to contain important SNPs, i.e. rs4803419 and rs3745274, among five major ethnic groups of the Pakistani population. Methods: Arlequin v3.5.DnaSPv6.12. and network 5 resources were used to analyze population genetic variance in the partial CYP2B6 gene sequences obtained from 104 human samples belonging to Punjabi, Pathan, Sindhi, Seraiki and Baloch ethnicities of Pakistan. The partial CYP2B6 gene region analyzed in the current study is previously known to possess important SNPs. Results: The data analyses revealed that genetic variance among samples mainly came from differentiation within the ethnic groups. However, significant genetic variation was also found among the various ethnic groups. The high pairwise Fst genetic distinction was observed between Seraiki and Sindhi ethnic groups ( $F s t=0.13392$, P-value $=0.026$ ) as well as between Seraiki and Balochi groups (Fst $=0.04303$, P-value $=-0.0030)$. However, the degree of genetic distinction was low between Pathan and Punjabi ethnic groups. Some SNPs, including rs3745274 and rs4803419, which are previously shown in strong association with increased plasma Efavirenz level, were found in high frequency. Besides, a novel SNP, which was not found in dbSNP and Ensemble databases, was identified in the Balochi ethnicity. This novel SNP is predicted to affect the CYP2B6 splicing pattern. Conclusion: These results may have significant implications in Pakistani ethnicities in the context of drugs metabolized by 
CYP2B6, especially in Seraiki and Balochi ethnicity. The novel heterogeneous SNP, found in the present study, might lead to altered drug-metabolizing potential of CYP2B 6 and, therefore, may be implicated in non-responder phenomenon. 
1 Inter-ethnic genetic variations and novel variant identification in the partial sequences of

4 Authors: Sagheer Ahmed*1, Hizbullah Khan*1, Asifullah Khan², Muhammad Hanif Bangash ${ }^{3}$, 5 Abrar Hussain ${ }^{4}$, Mughal Qayum ${ }^{5}$, Muhammad Hamid Hamdard ${ }^{6}$

$7{ }^{1}$ Department of Basic Medical Sciences, Shifa College of Pharmaceutical Sciences, Shifa Tameer8 e-Millat University, Islamabad, Pakistan.

9 2Department of Biochemistry, Abdul Wali Khan University Mardan (AWKUM), Pakistan

10 3Pakistan Institute of Nuclear Science \& Technology, Islamabad, Pakistan

$11{ }^{4}$ Balochistan University of Information Technology, Engineering \& Management Sciences, 12 Quetta, Pakistan

13 5 Department of Pharmacy, Kohat University of Science \& Technology, Kohat-26000, Pakistan

$14{ }^{6}$ Faculty of Biology, Kabul University, Afghanistan

15 Corresponding Author Name, Email, and Postal Address:

16 Dr. Muhammad Hamid Hamdard

17 Email; hamid.hamdard@,ku.edu.af,

18 Address: Kabul University, Kart-e-Char, Kabul, Afghanistan

Running title: Inter-ethnic variation in $C Y P 2 B 6$ in Pakistan 


\section{ABSTRACT}

26 Background: Some single nucleotide polymorphisms (SNPs) in the cytochrome P450 (CYP)2B6

27 gene lead to decreased enzyme activity and have an impact on drug metabolism. The present study

28 was designed to investigate the patterns of genetic distinction across a hypervariable region of the

29 CYP2B6 gene, known to contain important SNPs, i.e. rs4803419 and rs3745274, among five major

30 ethnic groups of the Pakistani population.

31 Methods: Arlequin v3.5.DnaSPv6.12. and network 5 resources were used to analyze population

32 genetic variance in the partial CYP2B6 gene sequences obtained from 104 human samples

33 belonging to Punjabi, Pathan, Sindhi, Seraiki and Baloch ethnicities of Pakistan. The partial

34 CYP2B6 gene region analyzed in the current study is previously known to possess important SNPs.

36 Results: The data analyses revealed that genetic variance among samples mainly came from

37 differentiation within the ethnic groups. However, significant genetic variation was also found among the various ethnic groups. The high pairwise Fst genetic distinction was observed between Seraiki and Sindhi ethnic groups $(\mathrm{Fst}=0.13392$, P-value $=0.026)$ as well as between Seraiki and Balochi groups $(\mathrm{Fst}=0.04303, \mathrm{P}$-value $=-0.0030)$. However, the degree of genetic distinction was

41 low between Pathan and Punjabi ethnic groups. Some SNPs, including rs3745274 and rs4803419, 42 which are previously shown in strong association with increased plasma Efavirenz level, were 43 found in high frequency. Besides, a novel SNP, which was not found in dbSNP and Ensemble 44 databases, was identified in the Balochi ethnicity. This novel SNP is predicted to affect the 45 CYP2B6 splicing pattern. 
46 Conclusion: These results may have significant implications in Pakistani ethnicities in the context

47 of drugs metabolized by CYP2B6, especially in Seraiki and Balochi ethnicity. The novel

48 heterogeneous SNP, found in the present study, might lead to altered drug-metabolizing potential

49 of $C Y P 2 B 6$ and, therefore, may be implicated in non-responder phenomenon.

50 Keyword: Pakistan, population genetics, pharmacogenetics, CYP2B6, Punjabi, Pathan, Sindhi,

51 Seraiki, Balochi.

52 INTRODUCTION

53 The cytochrome P450 2 (CYP2) is a major family of the cytochrome enzymes, comprising of

54 several subfamilies that aggregated together in the form of clusters in the genome. The CYP2

55 subfamilies include the $C Y P 2 A, C Y P 2 B, C Y P 2 F, C Y P 2 G, C Y P 2 S$ and $C Y P 2 T$ genes (Simonsson

56 et al., 2003). Among these, the $C Y P 2 B$ located at chromosome 19 of the human genome, holding

579 exons encodes for 491 amino acids containing protein (Yamano et al., 1989). The human

$58 C Y P 2 B 6$ gene has 2 known loci: the functional $C Y P 2 B 6$ and its non-functional pseudogene

$59 C Y P 2 B 7$, located in the center of the CYP2A18P locus inside a $112 \mathrm{~kb}$ block (Yamano et al.,

60 1989). The $C Y P 2 B 6$ enzyme is involved in the metabolic activation and inactivation of several

61 drugs including anticancer such as cyclophosphamide and ifosfamide (Chang et al., 1993; Granvil

62 et al., 1999; Roy et al., 1999), antimalarial such as artemesinin (Simonsson et al., 2003), antiviral

63 such as efavirenz, and antidepressant such as bupropion (Faucette et al., 2000). Although the total

64 fraction of the $C Y P 2 B 6$ enzyme is small as compared to the total hepatic P450 family, however, it

65 metabolizes a vast majority of pharmaceutical drugs. In addition to $7-8 \%$ of the marketed

66 pharmaceutical drugs, $C Y P 2 B 6$ metabolizes certain exogenous and endogenous substances such

67 as nicotine and testosterone (Rosenbrock et al., 1999), in conjunction with other cytochrome 68 enzymes. 
70 The Single nucleotide polymorphisms (SNPs) in the CYP2B6 gene may affect the expression and 71 enzyme activity of the translated protein, resulting in significant differences in the 72 pharmacokinetics of $C Y P 2 B 6$-metabolized drugs among individuals and ethnicities, in turn, 73 leading to variations in efficacy and toxicity (Desta et al., 2007; Nyakutira et al., 2008; Aurpibul 74 et al., 2012). Several important variants of $C Y P 2 B 6$ gene, i.e. CYP2B6*2 (C64T), *3 (C777A),

*4 (A785G), *5 (C1459T), *6 (G516T and A785G), *7 (G516T, A785G, and C1459T) (Lang et al., 2001), *8 (A415G) and *9 (G516T) have been discovered (Lamba et al., 2003), besides the wildtype $C Y P 2 B 6^{*} 1$ allele. About 38 protein variants of the highly polymorphic $C Y P 2 B 6$ gene have been identified so far (http://www. cypalleles.ki.se/CYP2B6.htm), (Zanger \& Klein, 2013). Genetic variants of the enzyme result in substrate, and expression dependent functional changes.

Numerous other studies have reported that genetic variations that alter the $C Y P 2 B 6$ enzyme expression result in altered drug responses (Hesse et al., 2000; Coller et al., 2002; Lerman et al., 2002). Studies have also shown that there are major differences in the amount of enzyme and its activity amongst different individuals. Ethnicity and sex-based differences in $C Y P 2 B 6$ gene expression and splice variants have been reported previously (Lamba et al., 2003). For example, CYP2B6 *4 variant has been shown to result in enhanced expression and altered activity of the enzymes (Gadel, Friedel \& Kharasch, 2015). A SNP rs3745274 (c.516G>T) leading to $C Y P 2 B 6^{*} 6$ allele was alone responsible for aberrant splicing, resulting in a low-CYP2B6 expression phenotype. In recent years, studies have identified $C Y P 2 B 6^{*} 6$ in association with enhanced plasma concentrations of certain drugs (Aurpibul et al., 2012), including efavirenz. 
92 Pakistan is ethnically diverse; however, little is known about the distribution of CYP2B6 genetic

93 variations in a country of over 210 million population. Various parts of the country possess a 94 unique lifestyle, diverse genetic background, dietary habits, culture, and geographical 95 environment. The five ethnicities focused on in the current study are the largest genetically and 96 linguistically distinct ethnicities of Pakistan. Among these, the Punjabi is the largest one residing

97 in the Punjab province. Geographically the area of Punjab is comprised of 98 eastern Pakistan and northern India. The Sindhi ethnicity primarily resides in Sindh province 99 located in Southeast of Pakistan. Pathan ethnic population is about 15 to $18 \%$ of total Pakistani 100 population. Pathan are predominantly residing in Khyber Pakhtunkhwa province situated in 101 northwestern region of Pakistan. Balochi people are residing predominantly in 102 Balochistan province situated in the South and Western region of Pakistan. The Saraiki people live 103 in Southern Punjab and Southern Khyber Pakhtunkhwa. The Saraiki people are distinguished in 104 culture and norms from the closely residing Punjabi and Pathan ethnic groups. Previously 105 published studies about these Pakistani population ethnic groups inferred considerable genetic 106 variation (Qamar et al., 2002; Quintana-Murci et al., 2004; Bergström et al., 2020). Furthermore, 107 distinct genetic ancestry of these groups has also been reported in worldwide population genetics 108 project (Li et al., 2008). The CYP2B6 gene contains many SNPs in addition to some copy number 109 variations. However, only a few might alter enzyme activity or be associated with certain diseases.

110 Keep in view this scenario, we investigated a part of $C Y P 2 B 6$ from the individuals belonging from

111 five major ethnic groups of Pakistan to infer their genetic composition across this important drug112 metabolizing enzyme.

\section{METHODS \& MATERIALS}




\section{Sample collection and DNA extraction}

116 The study includes 104 healthy human participants belonging to five different ethnicities of

117 Pakistan. There were 25 samples from Pathan ethnicity, 24 from Punjabi, 20 from Sindhi, 18 from

118 Seraiki, and 17 samples were from the Baloch ethnicity. The participants self-declared their

119 ethnicities. Ethical approval for the study was obtained from the Institutional Review Board \&

120 Ethics Committee of Shifa International Hospital/Shifa Tameer-e-Millat University, Islamabad.

121 Consent forms, both in English and Urdu (national language) were also approved by the same

122 committee. Urdu is understood throughout Pakistan. For DNA extraction, 5 milliliters of venous

123 blood was collected in EDTA tubes and stored in a refrigerator. DNA extraction kits

124 (ThermoScientific) wer used to isolate the genomic DNA from the blood samples. The quantity

125 and quality of the isolated DNA was analyzed by running it on a $1 \%$ agarose gel and by using

126 ultraviolet spectrophotometer at $260 \mathrm{~nm}$ and $280 \mathrm{~nm}$ wavelength. The ratio for most samples was

127 found in the range of $1.80 \pm 0.05$. Isolated genomic DNA was stored at $-20^{\circ} \mathrm{C}$ until further 128 processing.

129

130 DNA sequences and PCR amplification

131 The $C Y P 2 B 6$ target region holding important and significant genetic variants was amplified from

132 the purified genomic DNA. The PCR products were purified and submitted for commercial DNA

133 sequencing services to Tisngka Biotechnology, China. Each of the samples was sequenced in

134 triplicate $(3 \mathrm{x})$ for sequence validation. 
136 Data quality check and filtration

137 The quality of the DNA sequences was checked using the Staden package and Finch TV v1.4

138 (Geospiza, Inc; Seattle, WA, United States). High-quality sequence reads were assembled using

139 the Lasergene v7.1 package (DNASTAR Inc UA) as described previously (Hizbullah et al., 2020)

\section{Population Genetic Analyses}

142 The high-quality draft sequences were compared with the Human reference genome (GRch38)

143 coordinates deposited in the ensemble database. The SNPs positions were identified and scanned

144 against the genome-wide association study (GWAS) database. The GWAS database holds specific

145 markers reported in association with a particular trait. Besides, comparative analyses were

146 performed against different worldwide populations genetic variants data acquired from the 1000

147 Genome database (Auton et al., 2015) using Ferret v1.1 tool. The DnaSP v6.0 package was used

148 to calculate the sequence composition of parsimony informative sites and haplotypes. The

149 Arlequin v3.5 software was used to find out the population genetic statistics like Fst analysis

150 (pairwise fixation index), analysis of molecular variance (AMOVA), frequencies of haplotype,

151 and Nei's distance ' $\mathrm{D}_{\mathrm{A}}$ ' (to estimate the nucleotide differences among the population). The

152 haplotypes network plot was developed using the median-joining method introduced in the 153 NETWORK 10.0 package.

\section{Novel SNP assessment}

156 The functional prediction of the novel SNP was assessed by different in silico tools, including

157 MutationTaster-2, PolyPhen2, SIFT, and PredictSNP2. These tools can predict the non- 
158 synonymous and synonymous mutation of the coding and non-coding regions. PredictSNP2 was

159 used to find out deleterious, neutral, and unknown mutations. This resource provides a predictive

160 score based on various parameters like combine annotation dependent depletion (CADD),

161 deleterious annotation neural networks (DANN), computational framework for annotation and

162 prioritization in coding and non-coding region (FunSeq2), genome-wide annotation of variants

163 (GWAVA), fitness consequence (FitCons), and functional analysis through hidden Markov

164 models (FATHAMM).

\section{Data Availability}

166 The DNA sequences data generated in this study are available from GenBank under the following

167 accession number MW017225-MW017328.

\section{RESULTS}

\section{CYP2B6 Polymorphisms}

170 High-quality sequenced data of $C Y P 2 B 6$ was generated for 104 individuals belonging from major

171 ethnic groups of the Pakistani population. The target region was spanning genomic coordinates,

172 i.e. 19:41006882-41007421 of the Human reference genome GRch38. The sequenced data

173 completely covered the exon 4 and intron 4 and partially covered the third intron region of the

174 CYP2B6 gene. The data analysis in the context of human reference genome GRch38 identified

175 total three polymorphic loci. The annotations and allele frequencies of all the three polymorphic

176 loci are given in Table 1. The two observed SNPs, i.e. rs4803419 and rs3745274 are previously

177 reported in association with the drug response to efavirenz and/ or abacavir treatment. A single

178 heterozygous polymorphic locus was identified at the intronic-exonic border at position chr19:

$17941007072 \mathrm{G} \rightarrow \mathrm{A}$ (Figure S1). This SNP was not found in the Ensemble and dbSNP databases and

180 may be a novel SNP. During the functional annotation analyses the SNP showed no change in the 
181 primary amino acid sequence of the protein. However, the MutationTaster-2 resource identified

182 that this polymorphism might bring a change at the splicing site or enzyme activity, while the

183 PredictSNP2 predicted it as a neutral variant. This heterozygous polymorphic locus was found in

184 a Balochi individual with minimal allele frequency of 0.05882 (Table 1).

\section{Population Structure analysis}

186 The Fixation index (Fst) statistic was employed to investigate the genetic composition across

187 CYP2B6 gene among ethnic groups' samples. The high pairwise Fst genetic distinction was

188 observed between Seraiki and Sindhi ethnic groups $(\mathrm{Fst}=0.13392$, P-value $=0.026)$ as well as

189 between Seraiki and Balochi groups $(\mathrm{Fst}=0.04303, \mathrm{P}-\mathrm{value}=-0.0030)$. However, the degree of

190 genetic distinction was low between Pathan and Punjabi ethnic groups (Figure 1). Moreover, when

191 these ethnic groups were compared with the 1000 Genome samples, the Seraiki population showed

192 high genetic distinction $(\mathrm{P}$-value $=<0.01)$ in comparison with the East Asian: Han Chinese $(\mathrm{CHB})$,

193 Japanese (JPT); South Asian: Bengali (BEB), Sri Lankan Tamil (STU), Indian Telugu (ITU),

194 Gujrati Indian (GIH); and European populations: CEU. (Utah Residents CEPH with Northern and

195 Western European Ancestry) (Figure 2).

196

197 AMOVA was used to estimates the genetic deviation at a molecular level among and within

198 various ethnic groups samples. The significance of AMOVA was assessed by 99,000 permutations

199 (Table 2). The AMOVA model revealed that genetic diversity due to variations among-ethnic

200 groups is $1.08 \%$, and within-ethnic groups is $98.92 \%(\mathrm{P}$-value $=0.28615)$. Similarly, a variance

201 factor (Est. Var) of 0.50731 was observed within population; however, among-ethnic groups

202 variance factor was found low 0.00554. The among-ethnic group based genetic variance in this

203 model was although low but still significant, suggesting that ethnic groups are genetically distinct. 
204 High pairwise nucleotide differences $\left(D_{A}\right)$ were observed between Seraiki and Sindhi, Seraiki and 205 Balochi ethnic groups.

206

207 Similarly, a high mean pairwise difference $(\pi x y)$ was found in the Seraiki and Sindhi, Seraiki, and 208 Balochi ethnic groups. The highest within-population genetic distinction (i.e., $\pi$ ) was observed for 209 Balochi ethnic group samples (Figure S2)Error! Reference source not found.. Moreover, 210 significant differentiation between Seraiki and other Asian/European populations was found as 211 evident by the Nei's distance $\left(\mathrm{D}_{\mathrm{A}}\right)$ during comparison of Pakistani ethnicities CYP2B6 SNPs with

212 additional Asian and European populations data acquired from the 1000 Genome project (Figure $2133)$.

\section{Haplotypes composition}

216 A total of five haplotypes with haplotype diversity (Hd) of 0.7276 were identified for all the 104 217 sample sequences. The haplotype-1 was found with predominant frequency in Sindhi, Pathan, 218 Punjabi, and Balochi samples. The haplotype-2 was also found in the same ethnic groups including 219 Seraiki, whereas, haplotype-3 was found at high frequency only in the Seraiki group (Figure S3). 220 The AMOVA analysis of the pairwise distance between haplotypes revealed that Seraiki and 221 Sindhi predominant haplotype-2 showing substantial genetic differentiation against haplotype-5 222 (i.e., predominant in Balochi samples) and haplotype-1 (i.e., predominant in Sindhi and Balochi).

223 Moreover, the haplotype-3 (i.e. predominant in Seraiki samples) showing significant genetic 224 distinction against haplotype-5 and haplotype-4 (predominant in Balochi and Sindhi samples 225 (Figure 4). During medium-joining haplotype networking analysis, the main haplotypes of 226 Punjabi, Pathan, Sindhi, and Seraiki ethnic groups persisted on common nodes, whereas the 
227 Balochi samples predominant haplotype-5 constituted a separate node, showing the least genetic

228 connectivity with the rest of the ethnic groups (Figure 4).

229

230 DISCUSSION

231 The DNA sequencing approach can be implemented effectively to assess the allelic distribution of

232 the human DNA sequences and to estimate interethnic genetic variation among populations. This

233 approach has been frequently implemented across the world to identify specific individual

234 polymorphisms. The Pakistani population is a heterogeneous admixture and exhibits high ethnic

235 diversity due to complex demography and various invasions, including Central Asian, Arabs and

236 British colonial invasions (Bhatti et al., 2017). Therefore, allelic distribution-based assessment of

237 the drug-metabolizing enzymes coding genes, including CYP2B6 in different ethnicities of the

238 Pakistani population is expected to be a complex but an interesting project to study.

240 Current study provides the genetic structure of the CYP2B6 gene in major ethnic groups across the

241 Pakistani population. The enzyme encoding from $C Y P 2 B 6$ is responsible for the metabolism of

242 various pharmacological drugs including efavirenz (Ward et al., 1991). The polymorphisms

243 present in the $C Y P 2 B 6$ gene showed a distinction of allelic frequencies and genetic variability

244 among Pakistani ethnic groups. These differences may lead to variations in the drug-metabolizing

245 potential of the $C Y P 2 B 6$ enzyme. The former global population genomics projects like Human

246 Genome Diversity Project — HGDP (Auton et al., 2015; Bergström et al., 2020) identified genetic

247 distinction among various Pakistani ethnic groups. The various Pakistani population groups

248 (Indus-valley populations) revealing genetic patterns akin to the European, Caucasian, and Indian

249 populations (Metspalu et al., 2011). 
251 The determination of the various single nucleotide polymorphisms and allelic distribution of

252 human genes at a population level and their association with disease phenotypes have been

253 reported previously (Hamblin, Thompson \& Di Rienzo, 2002; Nyakutira et al., 2008). Several

254 genetic variants of $C Y P 2 B 6$ are reported to have a significant association with different drug

255 pharmacokinetics in multiple populations across the world, including African, Asian, and South

256 American (Klein et al., 2005; Nyakutira et al., 2008; Radloff et al., 2013). In the current study, we

257 observed significant genetic variation across $C Y P 2 B 6$ locus in Pakistani ethnicities in comparison

258 to worldwide, including South Asian, East Asian, and European populations. The pairwise

259 population genetics analyses of the data inferred less genetic differentiation among Punjabi,

260 Pathan, Balochi samples, while the Seraiki, Sindhi, and Balochi samples displayed high genetic

261 differentiation. The highest value of pairwise genetic differences was observed between the Seraiki

262 and Sindhi groups (Figure 1).

263

264 Interestingly the Seraiki group showed uniform genetic composition and the lowest intra-

265 population genetic variance (Figure S2). Therefore, we can expect a uniform phenotype in the

266 Seraiki population within the context of drugs metabolized by the CYP2B6 enzyme. The Seraiki

267 group seems to be distinct from the Punjabi ethnicity, despite these groups have close demography

268 as known from Bronze age Harappan civilization (the Pre-Aryan people) (Ahmed \& Khan;

269 Shackle, 1977; Hashmi \& Majeed, 2014).

270

271 The Balochi ethnicity showed higher intra and interethnic genetic differentiation, although this

272 group holds a small population size compared to the rest of Pakistani ethnicities. This 
273 heterogeneous genetic ancestry, as observed in the Balochi samples, may be due to their Aryan,

274 Arab, Persian, Turkish, Khurdish, Dravidian, Sewais (Hindu), and black African mixed ancestries

275 (Ahmed \& Khan). Therefore, due to high intraethnic genetic differentiation, the Balochi ethnic

276 group might exhibit distinct CYP2B6 mediated drug-metabolized and treatment response

277 phenotypes compare to other Pakistani ethnicities.

278

279 AMOVA, which was performed based on haplotypes significance analysis to determine the degree 280 of regional differentiation and homogeneity within and among Pakistani ethnic groups, found that 281 the majority of the genetic variance is attributed due to within-population factors. Genetic variance 282 among populations was only 1.08\%, as inferred during AMOVA analysis (Table 2). This 283 variability was due to the intra-ethnic group heterogeneity. The genetic variance between ethnic 284 groups in our study was low but is still considered significant as per previous studies (Adeyemo et 285 al., 2005). Nonetheless, the small genetic difference between ethnic groups variance does not 286 imply that they cannot be distinguished from each other. Instead, the pairwise analysis reveals 287 significance differences between the ethnic groups (Figure 1). Likewise, the results based on the 288 haplotypes composition (Figure S4) and haplotypes network plot (Figure 4) among ethnic groups 289 is congruent to the pairwise Fst analysis and further confirmed the genetic distinction among ethnic 290 groups.

292 The prevalence of $C Y P 2 B 6^{*} 6$ allele has been found variable throughout the world. Globally, $\mathrm{G}$ 293 allele frequency is 0.73 and that of T allele is 0.26 (Auton et al., 2015). In the present study, the 294 prevalence of $\mathrm{T}$ allele was found at 0.338 in the Pakistani population which is slightly lower than 295 its frequency found in the South Asian populations. Its prevalence in East Asia and Europe was 
296 found lower than Pakistan at 0.215 and 0.236 , respectively while its frequency was higher in

297 American and African populations, i.e. 0.373 and 0.374 respectively (Auton et al., 2015).

298

299 If clinicians have information about the patients' CYP2B6 gene, they may help enhance the efficacy

300 and reduce the adverse effect by prescribing the most suitable and safest drug to the patients based

301 on their genetic structure. Over 2.6 billion unit doses of drugs are dispensed each year in Pakistan

302 (Ahmed et al., 2020) and approximately 7.2\% marketed drugs are metabolized by CYP2B6

303 enzyme (Zanger \& Schwab, 2013). This means that over 187 million doses of those drugs are

304 dispensed annually in Pakistan which are metabolized by CYP2B6 enzyme. Our study shows that

305 about one third of Pakistan's population has a CYP $2 B 6^{*} 6$ allele. This implies that over 62 million

306 doses of drugs dispensed annually in Pakistan may not have desired effects as patients receiving

307 these medications possess a low activity CYP2B6 allele. For example, patients taking efavirenz

308 may suffer from enhanced frequency and severity of adverse effects if they possess one or two

$309 C Y P 2 B 6^{*} 6$ alleles. However, longitudinal studies with proper follow up are needed in the Pakistani

310 population to confirm these findings.

\section{CONCLUSION}

312 Overall, our results demonstrate distinct genetic patterns in different ethnic groups across Pakistan.

313 The novel heterogeneous SNP, as identified in the present study, might lead to altered drug-

314 metabolizing potential of CYP2B6 and, therefore, additional studies are required to decipher the

315 pharmacological impact of this SNP. The rs4803419, which has been previously shown to be

316 associated with increased plasma efavirenz level in different populations e.g., USA, Caucasian,

317 Black, and Hispanic (Holzinger et al., 2012) and Black South African populations (Pz et al., 2015),

318 was found in high frequency in Pakistani population. Likewise, the rs3745274 has also been 
319 previously shown in strong association with increased plasma Efavirenz level in African and

320 Serbian populations (A et al., 2014; Pz et al., 2015). This variant represents the $C Y P 2 B 6^{*} 6$ allele,

321 which decreases its enzymatic activity (Klein et al., 2005). This SNP, like rs4803419, is observed

322 in the current study for Seraiki, Sindhi, and Balochi samples with higher allelic frequencies

323 compared to other ethnic groups. These findings provide a foundation for future studies on the

324 mechanism and effects of CYP2B6 polymorphisms in the Pakistani population and are a step 325 towards personalized medicine.

327 Acknowledgments: The authors wish to thank Shifa Tameer-e-Millat University, Islamabad, for 328 providing an excellent academic environment to facilitate this kind of scholarly activity.

\section{REFERENCES}

332

333

334

335

336

337

A O, M S, A A, D J, J K, A O, G D. 2014.CYP2B6 516G>T (rs3745274) and smoking status are associated with efavirenz plasma concentration in a Serbian cohort of HIV patients. Available at https://pubmed.ncbi.nlm.nih.gov/24831655/ (accessed October 14, 2020). DOI: 10.1097/FTD.0000000000000098.

Adeyemo AA, Chen G, Chen Y, Rotimi C. 2005. Genetic structure in four West African population groups. BMC genetics 6:38. DOI: 10.1186/1471-2156-6-38.

Ahmed S, Altaf N, Ejaz M, Altaf A, Amin A, Janjua K, Khan AU, Imran I, Khan S. 2020. Variations in the frequencies of polymorphisms in the CYP2C9 gene in six major ethnicities of Pakistan. Scientific Reports 10:19370. DOI: 10.1038/s41598-020-76366-x.

Ahmed M, Khan G. The History of Baloch and Balochistan: A Critical Appraisal. :14. 
342 Aurpibul L, Chotirosniramit N, Sugandhavesa P, Kosashunhanan N, Thetket S, Supindham T,

343 Piyamongkol W, Supparatpinyo K. 2012. Correlation of CYP2B6-516G > T

344 Polymorphism with Plasma Efavirenz Concentration and Depression in HIV-Infected Adults in Northern Thailand. Current HIV research 10:653-660. DOI: $10.2174 / 157016212803901338$.

347

Auton A, Abecasis GR, Altshuler DM, Durbin RM, Abecasis GR, Bentley DR, Chakravarti A, Clark AG, Donnelly P, Eichler EE, Flicek P, Gabriel SB, Gibbs RA, Green ED, Hurles ME, Knoppers BM, Korbel JO, Lander ES, Lee C, Lehrach H, Mardis ER, Marth GT, McVean GA, Nickerson DA, Schmidt JP, Sherry ST, Wang J, Wilson RK, Gibbs RA, Boerwinkle E, Doddapaneni H, Han Y, Korchina V, Kovar C, Lee S, Muzny D, Reid JG, Zhu Y, Wang J, Chang Y, Feng Q, Fang X, Guo X, Jian M, Jiang H, Jin X, Lan T, Li G, Li J, Li Y, Liu S, Liu X, Lu Y, Ma X, Tang M, Wang B, Wang G, Wu H, Wu R, Xu X, Yin Y, Zhang D, Zhang W, Zhao J, Zhao M, Zheng X, Lander ES, Altshuler DM, Gabriel SB, Gupta N, Gharani N, Toji LH, Gerry NP, Resch AM, Flicek P, Barker J, Clarke L, Gil L, Hunt SE, Kelman G, Kulesha E, Leinonen R, McLaren WM, Radhakrishnan R, Roa A, Smirnov D, Smith RE, Streeter I, Thormann A, Toneva I, Vaughan B, Zheng-Bradley X, Bentley DR, Grocock R, Humphray S, James T, Kingsbury Z, Lehrach H, Sudbrak R, Albrecht MW, Amstislavskiy VS, Borodina TA, Lienhard M, Mertes F, Sultan M, Timmermann B, Yaspo M-L, Mardis ER, Wilson RK, Fulton L, Fulton R, Sherry ST, Ananiev V, Belaia Z, Beloslyudtsev D, Bouk N, Chen C, Church D, Cohen R, Cook C, Garner J, Hefferon T, Kimelman M, Liu C, Lopez J, Meric P, O’Sullivan C, Ostapchuk Y, Phan L, Ponomarov S, Schneider V, Shekhtman E, Sirotkin K, Slotta D, Zhang H, McVean GA, Durbin RM, Balasubramaniam S, Burton J, Danecek P, Keane TM, Kolb-Kokocinski 
A, McCarthy S, Stalker J, Quail M, Schmidt JP, Davies CJ, Gollub J, Webster T, Wong B, Zhan Y, Auton A, Campbell CL, Kong Y, Marcketta A, Gibbs RA, Yu F, Antunes L, Bainbridge M, Muzny D, Sabo A, Huang Z, Wang J, Coin LJM, Fang L, Guo X, Jin X, Li G, Li Q, Li Y, Li Z, Lin H, Liu B, Luo R, Shao H, Xie Y, Ye C, Yu C, Zhang F, Zheng H, Zhu H, Alkan C, Dal E, Kahveci F, Marth GT, Garrison EP, Kural D, Lee W-P, Fung Leong W, Stromberg M, Ward AN, Wu J, Zhang M, Daly MJ, DePristo MA, Handsaker RE, Altshuler DM, Banks E, Bhatia G, del Angel G, Gabriel SB, Genovese G, Gupta N, Li H, Kashin S, Lander ES, McCarroll SA, Nemesh JC, Poplin RE, Yoon SC, Lihm J, Makarov V, Clark AG, Gottipati S, Keinan A, Rodriguez-Flores JL, Korbel JO, Rausch T, Fritz MH, Stütz AM, Flicek P, Beal K, Clarke L, Datta A, Herrero J, McLaren WM, Ritchie GRS, Smith RE, Zerbino D, Zheng-Bradley X, Sabeti PC, Shlyakhter I, Schaffner SF, Vitti J, Cooper DN, Ball EV, Stenson PD, Bentley DR, Barnes B, Bauer M, Keira Cheetham R, Cox A, Eberle M, Humphray S, Kahn S, Murray L, Peden J, Shaw R, Kenny EE, Batzer MA, Konkel MK, Walker JA, MacArthur DG, Lek M, Sudbrak R, Amstislavskiy VS, Herwig R, Mardis ER, Ding L, Koboldt DC, Larson D, Ye K, Gravel S, The 1000 Genomes Project Consortium, Corresponding authors, Steering committee, Production group, Baylor College of Medicine, BGI-Shenzhen, Broad Institute of MIT and Harvard, Coriell Institute for Medical Research, European Molecular Biology Laboratory EBI, Illumina, Max Planck Institute for Molecular Genetics, McDonnell Genome Institute at Washington University, US National Institutes of Health, University of Oxford, Wellcome Trust Sanger Institute, Analysis group, Affymetrix, Albert Einstein College of Medicine, Bilkent University, Boston College, Cold Spring Harbor Laboratory, Cornell University, European Molecular 
of Medicine at Mount Sinai, Louisiana State University, Massachusetts General Hospital, McGill University, National Eye Institute N. 2015. A global reference for human genetic variation. Nature 526:68-74. DOI: 10.1038/nature15393.

391 Bergström A, McCarthy SA, Hui R, Almarri MA, Ayub Q, Danecek P, Chen Y, Felkel S, Hallast P, Kamm J, Blanché H, Deleuze J-F, Cann H, Mallick S, Reich D, Sandhu MS, Skoglund P, Scally A, Xue Y, Durbin R, Tyler-Smith C. 2020. Insights into human genetic variation and population history from 929 diverse genomes. Science 367. DOI: 10.1126/science.aay5012.

Bhatti S, Aslamkhan M, Attimonelli M, Abbas S, Aydın HH. 2017. Mitochondrial DNA variation in the Sindh population of Pakistan. DOI: 10.1080/00450618.2016.1144788.

Chang TK, Weber GF, Crespi CL, Waxman DJ. 1993. Differential activation of cyclophosphamide and ifosphamide by cytochromes P-450 2B and 3A in human liver microsomes. Cancer Research 53:5629-5637.

Coller JK, Krebsfaenger N, Klein K, Endrizzi K, Wolbold R, Lang T, Nüssler A, Neuhaus P, Zanger UM, Eichelbaum M, Mürdter TE. 2002. The influence of CYP2B6, CYP2C9 and CYP2D6 genotypes on the formation of the potent antioestrogen Z-4-hydroxy-tamoxifen in human liver. British Journal of Clinical Pharmacology 54:157-167. DOI: 10.1046/j.1365-2125.2002.01614.x.

Desta Z, Saussele T, Ward B, Blievernicht J, Li L, Klein K, Flockhart DA, Zanger UM. 2007. Impact of CYP2B6 polymorphism on hepatic efavirenz metabolism in vitro. Pharmacogenomics 8:547-558. DOI: 10.2217/14622416.8.6.547.

Faucette SR, Hawke RL, Lecluyse EL, Shord SS, Yan B, Laethem RM, Lindley CM. 2000. Validation of bupropion hydroxylation as a selective marker of human cytochrome P450 
411

412

413 Gadel S, Friedel C, Kharasch ED. 2015. Differences in Methadone Metabolism by CYP2B6 414

2B6 catalytic activity. Drug Metabolism and Disposition: The Biological Fate of Chemicals 28:1222-1230. Variants. Drug Metabolism and Disposition: The Biological Fate of Chemicals 43:9941001. DOI: $10.1124 / \mathrm{dmd} .115 .064352$.

Granvil CP, Madan A, Sharkawi M, Parkinson A, Wainer IW. 1999. Role of CYP2B6 and CYP3A4 in the in vitro N-dechloroethylation of (R)- and (S)-ifosfamide in human liver microsomes. Drug Metabolism and Disposition: The Biological Fate of Chemicals $27: 533-541$.

Hamblin MT, Thompson EE, Di Rienzo A. 2002. Complex Signatures of Natural Selection at the Duffy Blood Group Locus. The American Journal of Human Genetics 70:369-383. DOI: $10.1086 / 338628$

Hashmi R, Majeed G. 2014.Saraiki Ethnic Identity: Genesis of Conflict with State. Available at /paper/Saraiki-Ethnic-Identity\%3A-Genesis-of-Conflict-with-HashmiMajeed/e7906c679ad715aa871da0297ae45931d918d3dc (accessed October 14, 2020).

Hesse LM, Venkatakrishnan K, Court MH, von Moltke LL, Duan SX, Shader RI, Greenblatt DJ. 2000. CYP2B6 mediates the in vitro hydroxylation of bupropion: potential drug interactions with other antidepressants. Drug Metabolism and Disposition: The Biological Fate of Chemicals 28:1176-1183.

Hizbullah null, Ahmed S, Noor Mumtaz M, Zulfiqar Z, Amir Hamza S, Siraj S, Jelani M, Imran I, Khan A. 2020. Genetic variations in drug-metabolizing enzyme CYP2C9 among major ethnic groups of Pakistani population. Gene 746:144659. DOI: 10.1016/j.gene.2020.144659. 
434 Holzinger ER, Grady B, Ritchie MD, Ribaudo HJ, Acosta EP, Morse GD, Gulick RM, Robbins

435

436

437

438

439

440

441

442

443

444

445

446

447

448

449

450

451

452

453

454

455

GK, Clifford DB, Daar ES, McLaren P, Haas DW. 2012. Genome-wide association study of plasma efavirenz pharmacokinetics in AIDS Clinical Trials Group protocols implicates several CYP2B6 variants. Pharmacogenetics and genomics 22:858. DOI: 10.1097/FPC.0b013e32835a450b.

Klein K, Lang T, Saussele T, Barbosa-Sicard E, Schunck W-H, Eichelbaum M, Schwab M, Zanger UM. 2005. Genetic variability of CYP2B6 in populations of African and Asian origin: allele frequencies, novel functional variants, and possible implications for anti-HIV therapy with efavirenz. Pharmacogenetics and Genomics 15:861-873. DOI: 10.1097/01213011-200512000-00004.

Lamba V, Lamba J, Yasuda K, Strom S, Davila J, Hancock ML, Fackenthal JD, Rogan PK, Ring B, Wrighton SA, Schuetz EG. 2003. Hepatic CYP2B6 expression: gender and ethnic differences and relationship to CYP2B6 genotype and CAR (constitutive androstane receptor) expression. The Journal of Pharmacology and Experimental Therapeutics 307:906-922. DOI: 10.1124/jpet.103.054866.

Lang T, Klein K, Fischer J, Nüssler AK, Neuhaus P, Hofmann U, Eichelbaum M, Schwab M, Zanger UM. 2001. Extensive genetic polymorphism in the human CYP2B6 gene with impact on expression and function in human liver. Pharmacogenetics 11:399-415. DOI: 10.1097/00008571-200107000-00004.

Lerman C, Shields PG, Wileyto EP, Audrain J, Pinto A, Hawk L, Krishnan S, Niaura R, Epstein L. 2002. Pharmacogenetic investigation of smoking cessation treatment. Pharmacogenetics 12:627-634. DOI: 10.1097/00008571-200211000-00007. 
456 457

458

459

460

461

462

463

464

465

466

467

468

469

470

471

472

473

474

475

476

477

478

Li JZ, Absher DM, Tang H, Southwick AM, Casto AM, Ramachandran S, Cann HM, Barsh GS, Feldman M, Cavalli-Sforza LL, Myers RM. 2008. Worldwide Human Relationships Inferred from Genome-Wide Patterns of Variation. Science 319:1100-1104. DOI: 10.1126/science.1153717.

Metspalu M, Romero IG, Yunusbayev B, Chaubey G, Mallick CB, Hudjashov G, Nelis M, Mägi R, Metspalu E, Remm M, Pitchappan R, Singh L, Thangaraj K, Villems R, Kivisild T. 2011. Shared and Unique Components of Human Population Structure and Genome-Wide Signals of Positive Selection in South Asia. The American Journal of Human Genetics 89:731-744. DOI: 10.1016/j.ajhg.2011.11.010.

Nyakutira C, Röshammar D, Chigutsa E, Chonzi P, Ashton M, Nhachi C, Masimirembwa C. 2008. High prevalence of the CYP2B6 516G-->T(*6) variant and effect on the population pharmacokinetics of efavirenz in HIV/AIDS outpatients in Zimbabwe. European Journal of Clinical Pharmacology 64:357-365. DOI: 10.1007/s00228-007-0412-3.

Pz S, Pd L, Hm M, Pj S, Ja D, Ns L, G M, Dw H. 2015.Pharmacogenetics of plasma efavirenz exposure in HIV-infected adults and children in South Africa. Available at https://pubmed.ncbi.nlm.nih.gov/25611810/ (accessed October 14, 2020). DOI: 10.1111/bcp.12590.

Qamar R, Ayub Q, Mohyuddin A, Helgason A, Mazhar K, Mansoor A, Zerjal T, Tyler-Smith C, Mehdi SQ. 2002. Y-chromosomal DNA variation in Pakistan. American Journal of Human Genetics 70:1107-1124. DOI: 10.1086/339929.

Quintana-Murci L, Chaix R, Wells RS, Behar DM, Sayar H, Scozzari R, Rengo C, Al-Zahery N, Semino O, Santachiara-Benerecetti AS, Coppa A, Ayub Q, Mohyuddin A, Tyler-Smith C, Qasim Mehdi S, Torroni A, McElreavey K. 2004. Where West Meets East: The Complex 
479

480

481

482

483

484

485

486

487

488

489

490

491

492

493

494

495

496

497

498

499

500

mtDNA Landscape of the Southwest and Central Asian Corridor. American Journal of Human Genetics 74:827-845.

Radloff R, Gras A, Zanger UM, Masquelier C, Arumugam K, Karasi J-C, Arendt V, Seguin-Devaux C, Klein K. 2013. Novel CYP2B6 Enzyme Variants in a Rwandese Population: Functional Characterization and Assessment of In Silico Prediction Tools. Human Mutation 34:725-734. DOI: 10.1002/humu.22295.

Rosenbrock H, Hagemeyer CE, Singeç I, Knoth R, Volk B. 1999. Testosterone metabolism in rat brain is differentially enhanced by phenytoin-inducible cytochrome P450 isoforms. Journal of Neuroendocrinology 11:597-604. DOI: 10.1046/j.1365-2826.1999.00371.x.

Roy P, Yu LJ, Crespi CL, Waxman DJ. 1999. Development of a substrate-activity based approach to identify the major human liver P-450 catalysts of cyclophosphamide and ifosfamide activation based on cDNA-expressed activities and liver microsomal P-450 profiles. Drug Metabolism and Disposition: The Biological Fate of Chemicals 27:655-666.

Shackle C. 1977. Siraiki: A Language Movement in Pakistan. Modern Asian Studies 11:379-403. DOI: $10.1017 / \mathrm{S} 0026749 X 00014190$.

Simonsson USH, Jansson B, Hai TN, Huong DX, Tybring G, Ashton M. 2003. Artemisinin autoinduction is caused by involvement of cytochrome P450 2B6 but not 2C9. Clinical Pharmacology and Therapeutics 74:32-43. DOI: 10.1016/S0009-9236(03)00092-4.

Ward SA, Helsby NA, Skjelbo E, Brosen K, Gram LF, Breckenridge AM. 1991. The activation of the biguanide antimalarial proguanil co-segregates with the mephenytoin oxidation polymorphism-a panel study. British Journal of Clinical Pharmacology 31:689-692. DOI: 10.1111/j.1365-2125.1991.tb05594.x. 
501 Yamano S, Nhamburo PT, Aoyama T, Meyer UA, Inaba T, Kalow W, Gelboin HV, McBride OW, 502 Gonzalez FJ. 1989. cDNA cloning and sequence and cDNA-directed expression of human 503 P450 IIB1: identification of a normal and two variant cDNAs derived from the CYP2B 504 locus on chromosome 19 and differential expression of the IIB mRNAs in human liver. 505 Biochemistry 28:7340-7348. DOI: 10.1021/bi00444a029.

506 Zanger UM, Klein K. 2013. Pharmacogenetics of cytochrome P450 2B6 (CYP2B6): advances on 507 polymorphisms, mechanisms, and clinical relevance. Frontiers in Genetics 4:1-12. DOI: $508 \quad 10.3389 /$ fgene.2013.00024.

509 Zanger UM, Schwab M. 2013. Cytochrome P450 enzymes in drug metabolism: Regulation of gene 510 expression, enzyme activities, and impact of genetic variation. Pharmacology \& 511 Therapeutics 138:103-141. DOI: 10.1016/j.pharmthera.2012.12.007.

512 
Figure 1

Matrix of pairwise Fst-Pakistani Ethnicities

Heatmap: Pairwise Fst (Fixation index) among five ethnic groups of Pakistani population depicted high genetic differentiation between Seraiki and Sindhi. 


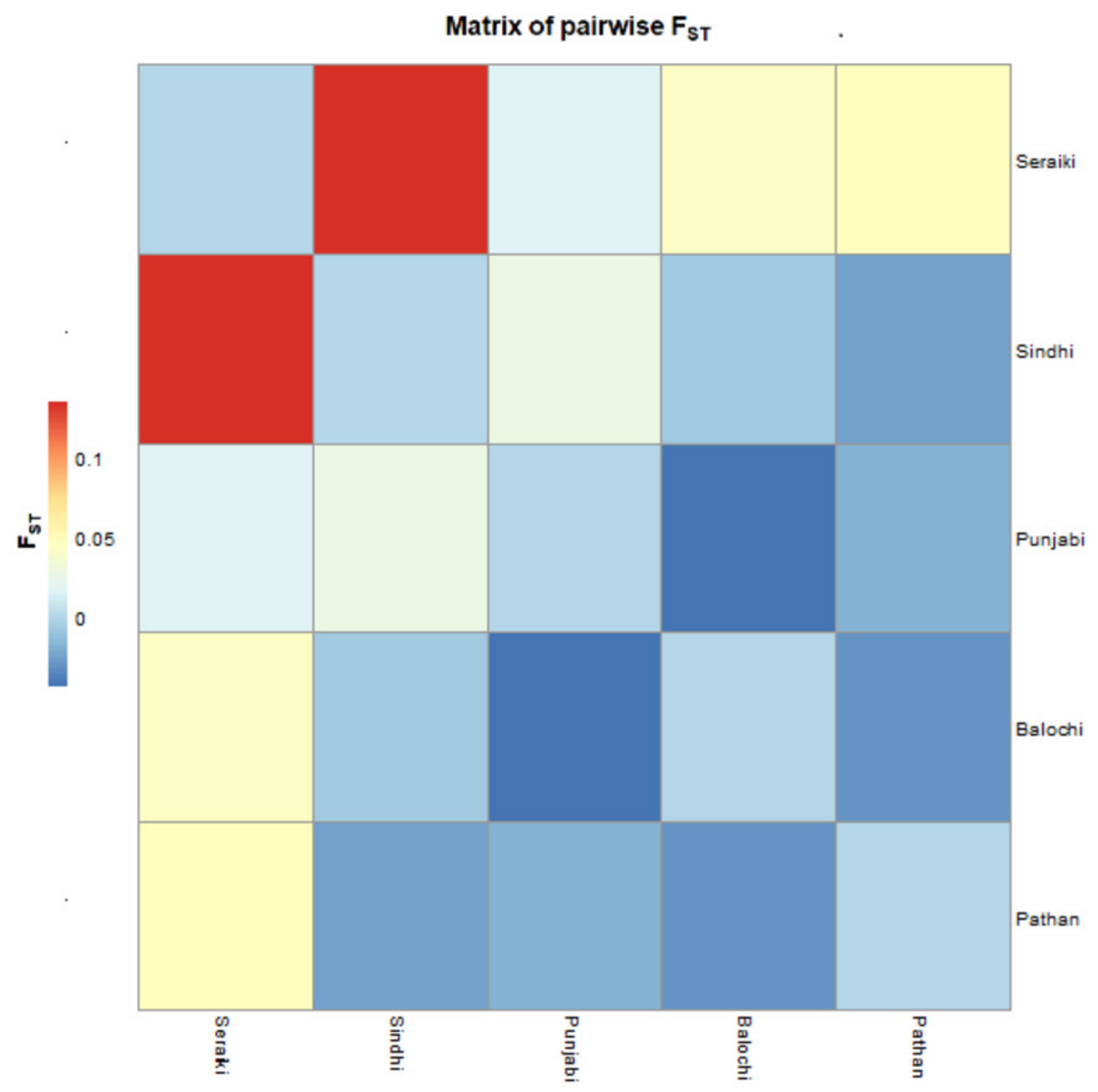


Figure 2

Matrix of pairwise Fst-Global populations

Pairwise Fst analysis in the Structure of other Asian Population i.e. BEB, GIH, ITU, STU, CHB, JPT, and European (CEU) population data compiled from 1000 Genome Project depicted high genetic differentiation with Seraiki individual followed by other ethnic groups.

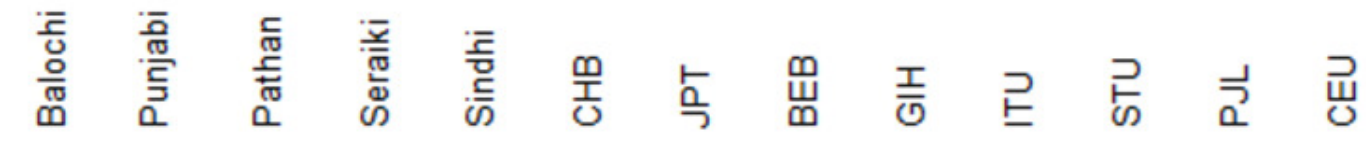

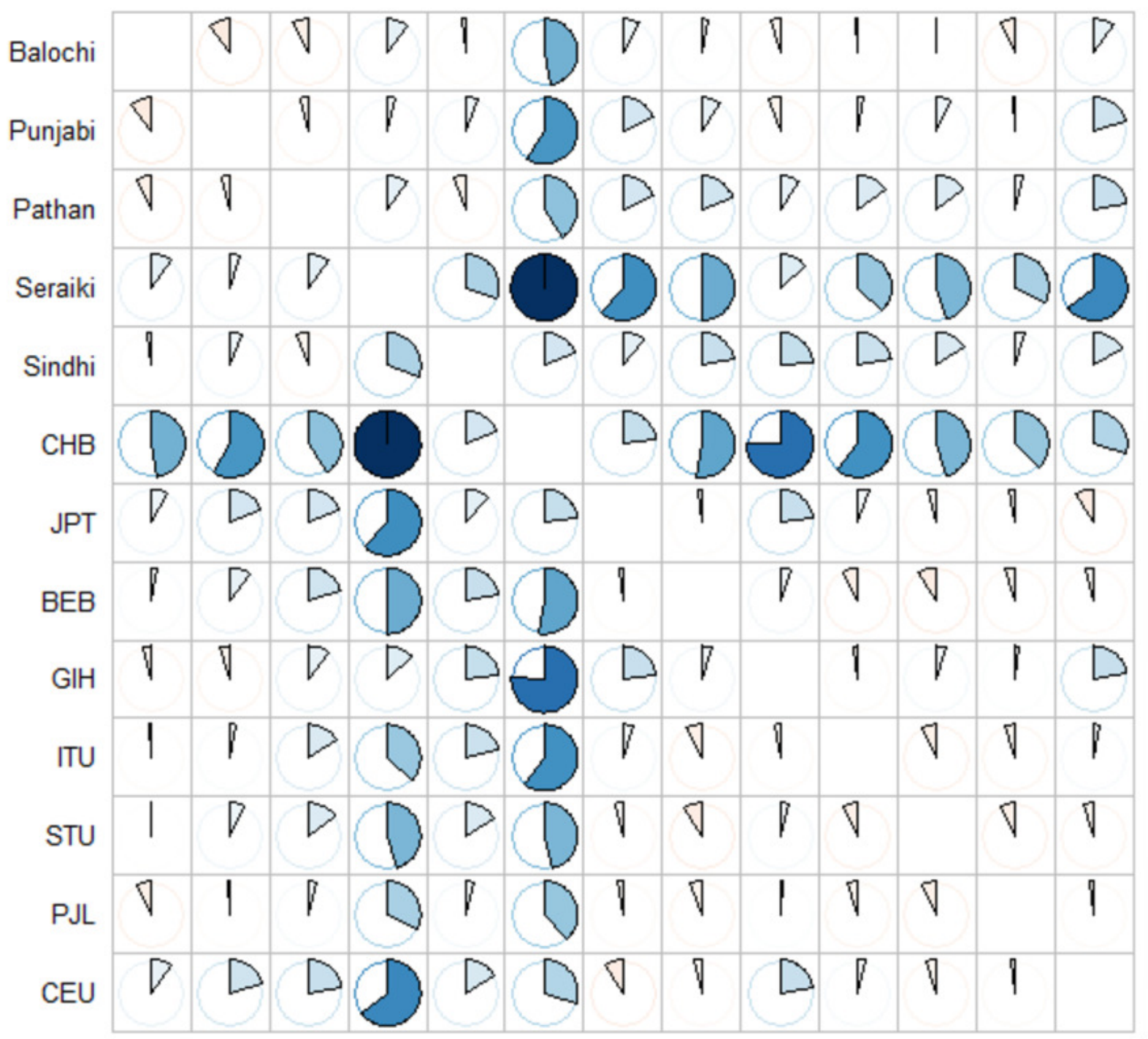




\section{Table 1 (on next page)}

Allele frequencies and annotations of the CYP2B6 polymorphism 
2 Table 1. Allele frequencies and annotations of the $C Y P 2 B 6$ polymorphism observed in five major 3 ethnic groups of the Pakistani population.

(1)

\begin{tabular}{|c|c|c|c|c|c|c|c|c|c|c|}
\hline \multirow[b]{2}{*}{ S/No } & \multirow[b]{2}{*}{ References } & \multirow[b]{2}{*}{ Position } & \multirow{2}{*}{$\begin{array}{c}\text { REF/ALT } \\
\text { Alleles }\end{array}$} & \multirow[b]{2}{*}{ Consequences } & \multirow{2}{*}{$\begin{array}{l}\text { Effect in } \\
\text { HGVS- } \\
\text { Nomenclature }\end{array}$} & \multicolumn{5}{|c|}{ Minor Allele Frequencies (\%) } \\
\hline & & & & & & Balochi & Punjabi & Pathan & Seraiki & Sindhi \\
\hline 1 & rs4803419 & $19: 41006887$ & $\mathrm{C} / \mathrm{T}$ & Intron variant & g.20589C>T & 47.058 & 45.833 & 60.000 & 55.555 & 65.000 \\
\hline 2 & rs3745274 & 19:41006936 & $\mathrm{G} / \mathrm{T}$ & $\begin{array}{l}\text { Missense } \\
\text { variant }\end{array}$ & Q172H & 47.058 & 54.166 & 48.000 & 77.777 & 35.000 \\
\hline 3 & Novel & 19:41007072 & $\mathrm{G} / \mathrm{G}, \mathrm{A}$ & Intron variant & g.20774G>A & 5.882 & No & No & No & No \\
\hline
\end{tabular}

5 References: SNP Id in dbSNP database; Position: Coordinate position in refence genome

6 (GRch38.p12 chr:19), REF: Reference allele; $\boldsymbol{A L T}$ : Alternate or variant allele; HGVS-

7 Nomenclature: of the Human Genome Variation Society to show the description of the sequence

8 variant (substitution, deletion, insertion, duplication etc.) 
Table 2 (on next page)

Analysis of genetic distinction across CYP2B6 
1

2 Table 1. Analysis of genetic distinction across $C Y P 2 B 6$ among and within ethnic groups samples

3 of Pakistani population.

\begin{tabular}{|l|l|l|l|l|l|}
\hline Source of variation & Df & SS. & Est. Var & $\%$ & P-value \\
\hline Among populations & 4 & 2.488 & 0.00554 & 1.08 & $<0.28615$ \\
\hline Within population & 99 & 50.244 & 0.50731 & 98.92 & $<0.28615$ \\
\hline
\end{tabular}

$4 d \boldsymbol{d}:$ degrees of freedom; $\boldsymbol{S S}$ : the sum of squares deviation; Est.Var: estimates of variance

5 components; \%: percentage of total variance contributed by each component. 


\section{Figure 3}

Average pairwise differences-Global populations

Pairwise comparison of Pakistani ethnic groups with other Asians populations i.e. -East Asian: BEB, GIH, ITU, STU.; South Asian: CHB, JPT and Europeans CEU. retrieved from 1000 Genome Browser, found that high genetic distinction between population (green above diagonal) of Seraiki, Sindhi as with the Nei's distance (blue below diagonal) followed by other Asian and European population. 


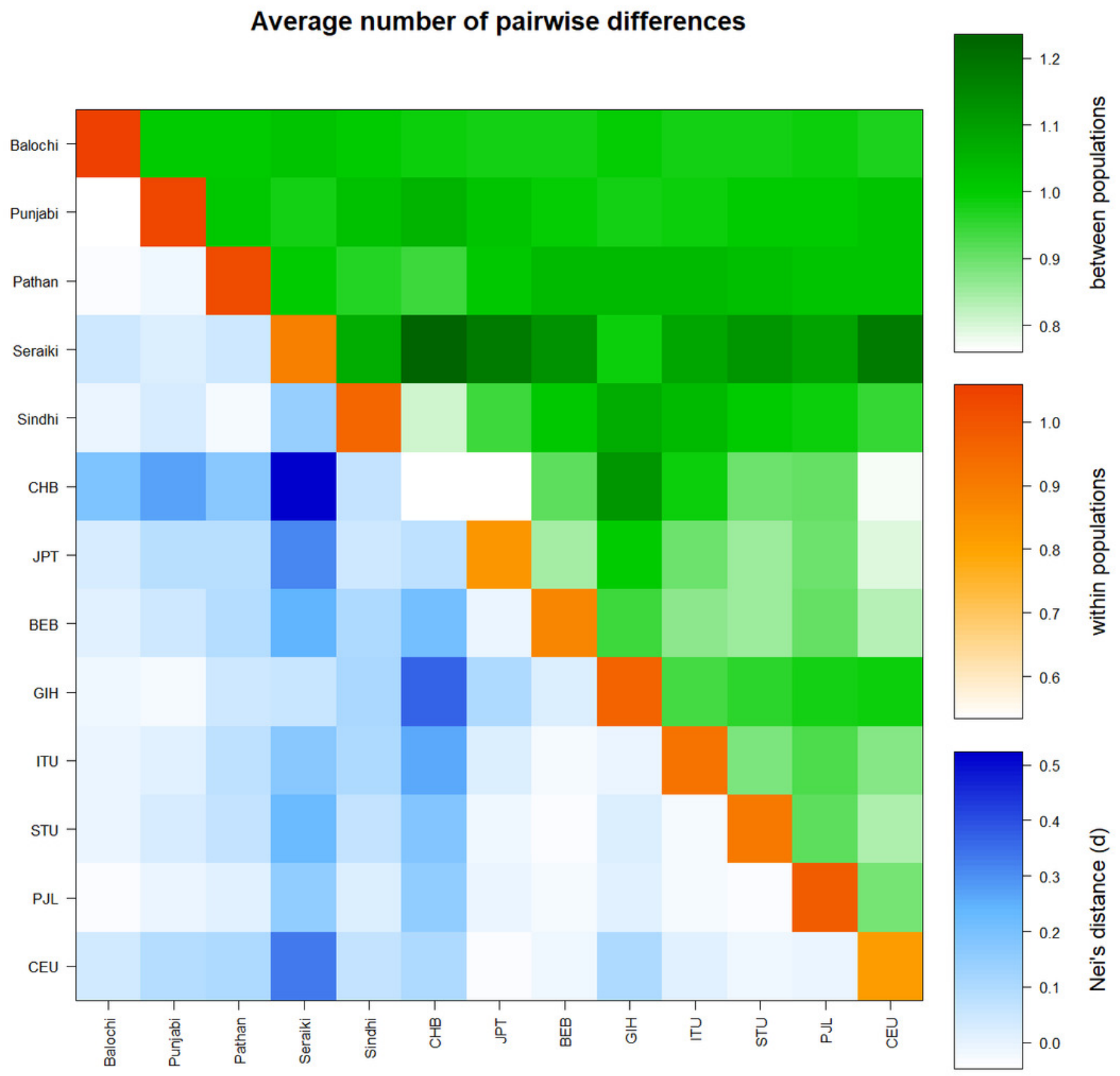


Figure 4

Haplotypes network

Haplotypes Network plot: Circle area are proportional to the number of taxa holding that particular haplotypes. The lines connecting the haplotypes reflect the distance of relatedness. While the colored show distinction between ethnic groups - Red: Balochi, Pansypurple: Punjabi, Yellow: Pathan, Blue: Seraiki, Green: Sindhi. 


\section{Haplotypes Network}

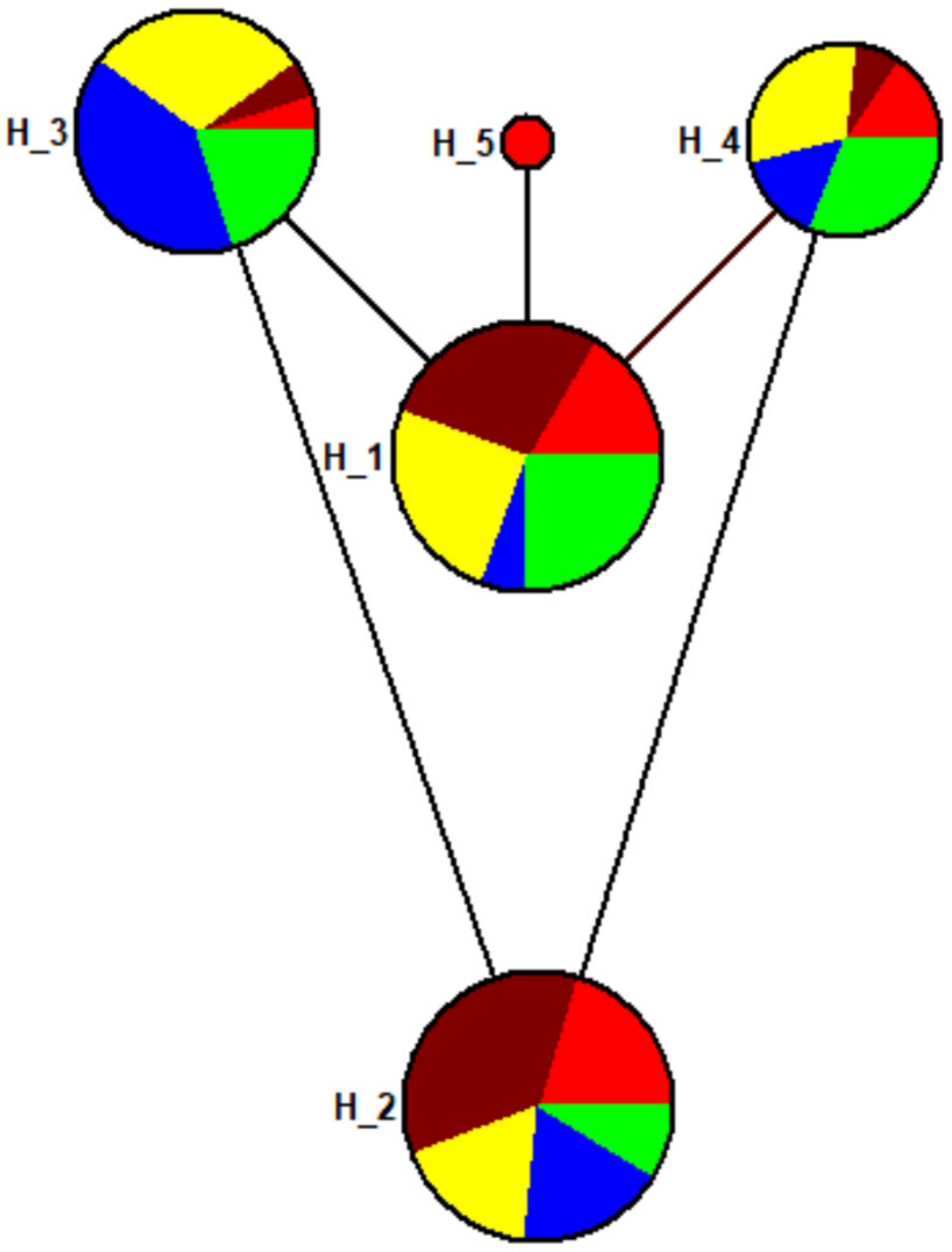

